Introducing CBT Self-Help Approaches into Condition Management Teams Sheraz Ahmed ${ }^{1}$, Anne Joice ${ }^{1}$ Catriona Kent $^{1}$, \& Chris Williams ${ }^{1}$

${ }^{1}$ University of Glasgow 


\title{
Introducing CBT Self-Help Approaches Into Condition Management Teams
}

\author{
Sheraz Ahmed, Anne Joice, Catriona Kent, Chris Williams \\ Introduction
}

The Government's welfare to work programmes have sought to improve the lives of people receiving out of work benefits by helping them move into work (Department for Work and Pensions (DWP) 26). The Green Paper 'Pathways to work: Helping people into Employment' (2002) outlined changes to the claiming requirements and the services offered to people making a new or repeat claim for Incapacity Benefit. The introduction of these new services has resulted in an $8 \%$ decrease in people continuing on Incapacity benefit (IB) in the Pilot sites for the new approach (DWP 26).

The key elements of the new provision include Incapacity Benefit Personal Advisors who perform work focused interviews, Return to Work Credit, and a Choices Package (DWP 346). Condition Management Programmes are an option of the Choices package and were developed jointly between Jobcentre Plus and local NHS providers.

The Condition Management teams were designed in response to the main conditions reported by those claiming Incapacity Benefit (mental health, musculoskeletal, and cardio-vascular). They adopt a bio-psychosocial model with an emphasis on enabling the customer to better manage their health condition. The aim is to improve both quality of life and employability (DWP 346). Being in employment offers many benefits to the individual, their families and to the wider society.

CMP teams are staffed by health professionals from a diverse range of physical and mental health backgrounds including nurses, physiotherapists, occupational therapists, health psychologists, and occupational health nurses. This brings advantages with the availability of a range of skills and outlooks. However, this very variety means there is no common language of assessment.

The Condition Management Programme targets both mental and physical health conditions. A new language of relationship is used to describe working with "customers" rather than "patients" or "clients" and the use of this language is partly chosen to emphasise that staff are not working in a health care setting. Between 2003 and 2005, 7490 referrals were made to the pilot Condition Management Programmes (CMP) (DWP 26). Overall, $80 \%$ of customers had mental health problems and many experienced complex difficulties (DWP 346). Incapacity Benefit Advisers have a tendency to refer cases to CMP that are "more difficult to help" and those individuals are "expected to take longer to get jobs" with only $30 \%$ of customers referred for condition management expecting to return to work within the next 6 months (DWP 26). Condition Management Practitioners therefore often face the challenge of working with complex cases 
who have low therapeutic optimism and may present with physical and/or mental health problems.

The desired emphasis on empowerment and collaboration, as opposed to treatment, make Cognitive Behavioural Therapy based interventions a key choice for service provision from several of the CMP sites. The evidence base for CBT for common mental health problems is identified in several NICE reports (e.g. anxiety, depression - NICE 2004 a, b) and Cochrane reviews of a wide range of physical health problems (www.cochrane.org/reviews). Typically Cognitive Behavioural Therapy has in the past traditionally only been available delivered by highly specialist practitioners in 12 to 20 one-hour sessions. This model of delivery creates problems with access and availability (Lovell and Richards 2000). Lovell and Richards argue that services that provide only "traditional" CBT are not evidence-based. Their article illustrates that CBT can also be successfully delivered in other ways such as groups, by telephone, and using CBT self-help. CBT self-help can be an effective intervention for depression and anxiety as part of low intensity high capacity services. The evidence base for the effectiveness for CBT self-help is good for depression and anxiety - however self-help is far more effective when delivered with support or guidance (Gellatly et al, 2007; Anderson et al, 2005). This is important because anxiety and depression are common primary problems in CMP referrals - and also may be present as a consequence of long-term physical health problems.

\section{The Five Areas Approach}

A key issue restricting delivery of CBT is the complex language often used (e.g. dysfunctional assumptions and negative automatic thoughts). The reading age required to use such words is over 19 (Williams and Garland 2000). Such terms are difficult for practitioners and customers to learn. In addition, traditional CBT courses are expensive in terms of both cost (often >£3500) and time. Additionally, access to such one year postgraduate courses is restricted as a result. The five areas series of CBT self-help workbooks was developed by $\mathrm{Dr}$ Chris Williams and was initially commissioned to produce an accessible form of CBT that was acceptable to a range of practitioners and customers (Williams, 2006). The language used is the most accessible of the most commonly offered CBT self-help books used on book prescription schemes and recommended by CBT practitioners (Martinez et al, in press). It aims to help both clinicians and customers discover why the person feels as they do and also to identify clear targets for change. Crucially the assessment is collaborative and jointly agreed therefore, engaging the customer in the process of change.

The five areas assessment model aims to provide a clear structure to summarise the range of problems and difficulties faced by the person in each of the following domains:

- Life situation, relationship and practical problems

- Altered thinking

- Altered emotions (mood or feelings)

- Altered physical feelings/symptoms in the body

- Altered behaviour or activity levels 
This provides a flexible assessment that can be personalised and tailored to the individual no matter what balance of psychological, social or physical problems they face. An example of the Five Areas Assessment model is shown in Diagram 1 which summarises the case of a 42-year-old married woman called Fiona who has recently lost her post as a teacher. It indicates that what a person thinks about a situation or problem may affect how they feel physically and emotionally, and also alters what they do (behaviour or activity). Each of these five areas (situation, relationship or practical problems, thinking, emotional and physical feelings, and behaviour changes) affects each other.

\section{Diagram 1: The Five Areas Assessment Model of Fiona - a 41 year old married mother of 2.}

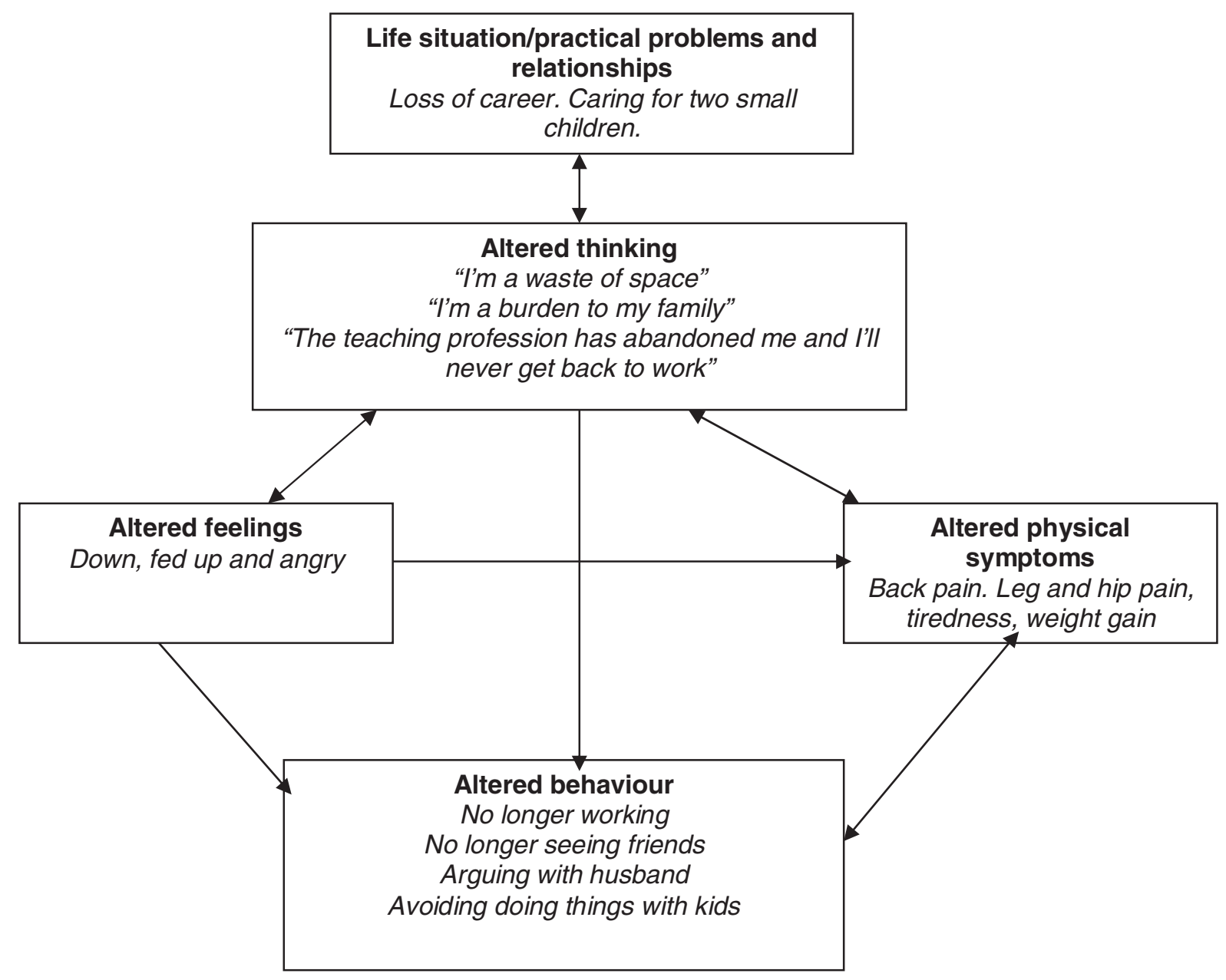

The Condition management team helped Fiona overcome her reduced activity such as seeing her friends by identifying this as a target and planning ways of reintroducing such activities in a planned step by step way. This also helped boost her mood and confidence. She became less argumentative and more engaged with her family. Over several months she became more positive about seeking employment and returned to doing supply work. 


\section{Using the Model in Assessment}

Area 1: Situation, relationship and practical problems: Many customers face practical problems and life difficulties both directly and indirectly associated with being off or out of work. The actions of people around them can create upsets and difficulties as well as being a potential source of support. Practical problems such as relationship issues or financial difficulties may also be present. Such problems may include debts, housing or other difficulties, problems in relationships with family, friends or colleagues, and life events and difficulties such as deaths, redundancy, divorce, and interviews with incapacity benefits advisors, etc.

Area 2: Altered thinking: At times of distress thinking may alter and become extreme and unhelpful. For example the person may become very biased against themselves and self-critical or be prone to jump to the conclusion that the very worst will happen. This altered thinking is important because what a person thinks can affect how they feel emotionally and unhelpfully alter what they do. For example, someone whose immediate thought when first referred to their condition management worker is "they'll just judge me" may well feel anxious and be tempted to avoid the situation.

Area 3: Altered mood: This may include feeling low, depressed or anxious in mood, or reflect feelings of guilt, shame or anger.

Area 4: Altered physical symptoms: These may include problems such as pain and restricted movement such as stiffness. In addition a range of altered symptoms related to stress or depressed mood may occur such as early morning wakening, disrupted sleep, poor appetite and fatigue in depression, and feeling sweaty, clammy, shaky and breathless in anxiety. It can also summarise any other symptoms directly caused by physical disease.

Area 5: Altered behaviour: Some altered behaviours may act to worsen or keep problems going. These may include:

a) Reduced activity: where the person reduces or stops doing things that previously gave a sense of achievement or pleasure. This occurs as a result of illness or low mood. For example, the person may stop meeting friends or reduce their hobbies/interests. The result is a vicious circle of reduced activity (Williams 2006) that worsens how they feel. A useful question to help identify the impact of reduced activity is "What things have you stopped doing since you started feeling like this?"

b) Areas of avoidance: When someone is anxious about illness, they will often reduce or stop doing things that seem to worsen their symptoms. This may lead to avoidance of different situations, people or places and result in a vicious circle of avoidance (Williams 2006). Useful questions to help identify the impact of avoidance are "What things have you stopped doing since you started feeling like this?" and "What would you be able to do if you weren't feeling this way?" 
c) Identifying unhelpful behaviours: People respond to distress in various ways. This might include helpful things such as talking to friends and relatives, or keeping active and involved in things. However sometimes the responses may backfire and become unhelpful. Examples might include excessive drinking, drug misuse, becoming very clingy and dependent, over-eating (comfort eating), risktaking or self-harming behaviours. The result is a vicious circle of unhelpful behaviour that worsens and worsens how they feel. A useful question to help identify the impact of unhelpful behaviours is "What things have you started doing to cope?"

A series of checklists and written summaries of these vicious circles are available for free download from www.livinglifetothefull.com in the resources area. This site also offers other health and employability resources including a live chat, the capacity for practitioners to monitor customer use of the site and mood rating scales as well as training resources for practitioners and customers.

In addition, workbooks to help people self-assess and self-manage key changes in each of the five areas are available (Williams 2007). The first edition of this book has recently been evaluated in a funded randomised controlled study and found to be very effective when compared to treatment as usual with improved mood at both four and twelve months on an intention to treat analysis, greater satisfaction than treatment as usual, and at a small cost saving compared to treatment as usual (Williams et al 2007).

\section{CMP Training Using the Five Areas Approach}

We have developed a course that teaches staff members how to work effectively using the five areas assessment and the linked workbooks The Five areas Condition Management Team training course provides skills based training that addresses both physical and mental health and promotes self-management. It aims to increase practitioner confidence and skills in using the Overcoming depression and low mood self-help workbooks (Williams 2006).

The training is delivered by experienced Cognitive Behavioural Therapists and provides an introduction to the Five areas model and it's practical application across a range of difficulties a customer might typically face. Customers facing both physical and mental health difficulties may frequently fall into patterns of behaviours. The skills incorporated into the training aim to help practitioners introduce their customers to skills to helpfully change these patterns.

These include:

- Identifying, assessing and overcoming vicious circles of Reduced Activity, Avoidance and Unhelpful Behaviours whilst also building more helpful responses

-Anxiety Control Training

- Practical Problem solving

-Identifying and Changing extreme and unhelpful thinking

- Healthy Living and Relapse Prevention Strategies 
Case examples include lower back pain, multiple sclerosis, anxiety, depression, flu and arthritis. Participants are also encouraged to utilise anonymised examples from their own caseloads, which are discussed in the teaching sessions.

Alongside the training practitioners have access to a range of written self help tools for use by customers, including how to use the www.livinglifetothefull.com website resource.

The aim of the training: is to increase the confidence of the attendees in using a psychosocial approach and self help materials based on Cognitive Behavioural Therapy.

Key features of the training include:

-A consistent accessibly language

- Linked supporting written materials and website information.

-Interactive workshops with experiments to test the approach out.

\section{We have evaluated the delivery of the training using two different delivery models:}

-Slow-track: Six half-day sessions were delivered over a three to four month period. This allowed practitioners to put what they were learning into practice. Each session started and ended with a practice and review component. Course participants are expected to utilise self directed learning using personal learning logs. Practical skills acquisition is aided by actors performing the role of mock customers.

- Fast-track: the 6 sessions were provided over a condensed three day intensive course delivered by the three trainers $(\mathrm{AJ} / \mathrm{CK} / \mathrm{CW})$. Here the components concerning supervision, practice and review were omitted and in contrast to the Slow Track training the course attendees had no time to return to routine work and try the approach out between sessions.

\section{Training Outcomes}

Team members were asked to complete and return questionnaires to evaluate their knowledge and skill before and after the course. These evaluated:

- Subjective knowledge and skill regarding key principles of cognitive behaviour therapy using a Likert scale of 1 (very poor) to 7 (excellent).

- Objective skill regarding key principles of CBT using self-test tasks.

-Subjective usefulness of this approach to Condition Management using a Likert scale of 1 (not at all useful) to 7 (extremely useful) 


\title{
Who Attended the Training?
}

115 practitioners have completed the five areas CMP in three sites. The following data is based on the 81 individuals who have handed back their baseline and final evaluations (Leeds, Glasgow and Liverpool).

Figure 1 summarises the professional background of course attendees. The average length of time that attendees were qualified in their profession was 14 years $(n=72)$.

\section{Figure 1: Attendees by Profession}

\section{Registered Profession}

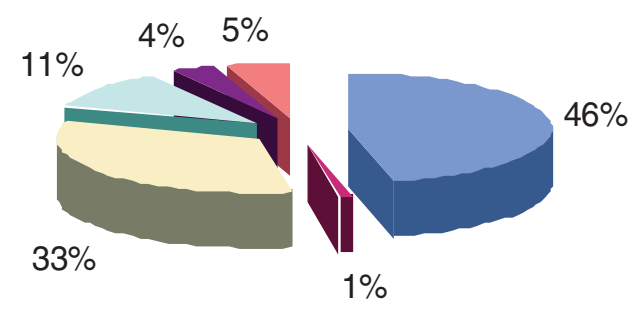

\author{
Nursing \\ - Social Work \\ Occupational Therapy \\ Physiotherapy \\ Clinical Psychology \\ Other
}

Overall, thirty staff members stated they had received previous training in cognitive behaviour therapy and 49 had not. The nature of this previous training is not known.

\section{Subjective Knowledge and Skill Gains}

Attendees were asked twenty questions regarding their subjective knowledge (10 questions) and skill (10 questions) about key aspects of cognitive behaviour therapy and self-help at baseline and on course completion, and were asked to rate themselves on a Likert scale of $1-7$ ( 1 = very poor; 7 = excellent).

\section{Objective Measures of Skill}

Objective measures of skill were measured using a practical clinical skills-based task. This involved identifying clinical symptoms and completing a problemfocused assessment.

Results for both the subjective knowledge and skills and objective skills tasks are summarised in Table 1. 

Table 1: Knowledge and Skill Gains Pre and Post Five Areas
Condition Management

\begin{tabular}{|c|c|c|}
\hline QUESTION & $\begin{array}{c}\text { SLOW TRACK } \\
(N=24) \\
\text { MEAN DIFFERENCE }\end{array}$ & $\begin{array}{c}\text { FAST TRACK } \\
(\mathrm{N}=\mathbf{2 4}) \\
\text { MEAN DIFFERENCE }\end{array}$ \\
\hline $\begin{array}{c}\text { Self Perceived } \\
\text { Knowledge Questions }\end{array}$ & $\begin{array}{c}\text { Mean } 37.78 \text { changing to } 53.13 \\
t=-12.4,15 \mathrm{df}, \mathrm{p}=0.000 \\
95 \% \mathrm{Cl}-17.988 \text { to }-12.712\end{array}$ & $\begin{array}{c}\text { Mean } 39.31 \text { changing to } 53.93 \\
t=-11.979,41 \mathrm{df}, \mathrm{p}=0.000 \\
95 \% \mathrm{Cl}-17.081 \text { to }-12.153\end{array}$ \\
\hline $\begin{array}{c}\text { Self Perceived } \\
\text { Ability/Skill Questions }\end{array}$ & $\begin{array}{c}\text { Mean } 39.26 \text { changing to } 52.24 \\
t=-12.400 .15 \mathrm{df}, p=0.000 \\
95 \% \mathrm{Cl}-15.572 \text { to }-10.369\end{array}$ & $\begin{array}{c}\text { Mean } 41.13 \text { changing to } 54.22 \\
t=-11.171 .40 \mathrm{df}, p=0.000 \\
95 \% \mathrm{Cl}-15.461 \text { to }-10.724\end{array}$ \\
\hline Objective Skills & $\begin{array}{c}\text { Mean } 85.33 \text { changing to } 91.2 \\
t=-3.561,24 d f, p=0.002 \\
95 \% \mathrm{Cl}-9.267 \text { to }-2.467\end{array}$ & $\begin{array}{c}\text { Mean } 84.39 \text { changing to } 84.23 \\
\mathrm{t}=0 / 040,40 \mathrm{df}, \mathrm{p}=0.968 \mathrm{~ns} \\
95 \% \mathrm{Cl}-8.007 \text { to } 8.333\end{array}$ \\
\hline
\end{tabular}

The clear difference here is that although self-perceived learning was similar in both teaching styles, there was no gain change in objective skill in the fast track attendees, compared to a very significant and important gain in skills in those attending the slow track course.

\section{Overall Course Satisfaction}

Attendees were asked to rate how useful the training had been on a scale of 1-7. The mean usefulness was found to be 5.8, representing a high level of usefulness. The course was rated as being well presented (mean 5.8 using a scale of 1-7 with 1 not at all well and 7 being extremely well) - representing a high level of satisfaction with the presentation. Overall, $98 \%$ of attendees stated that they would recommend the course to other condition management practitioners.

\section{Other Related Resources Available to Support Customers}

A range of other resources have been made available free of charge for use by the public. They include:

a) The www.livinglifetothefull.com (LLTTF) website. This is a free online cognitive behaviour therapy course offering cognitive behaviour therapy life skills training. The site has proved very popular with an average of over a million hits each month. The site uses the same approach and language as the course and materials and provides an additional helpful resource for CMP's. The website is winner of the 2007 Excellence in the Practice of Counselling and Psychotherapy awards given by the British Association for Counselling and Psychotherapy. 


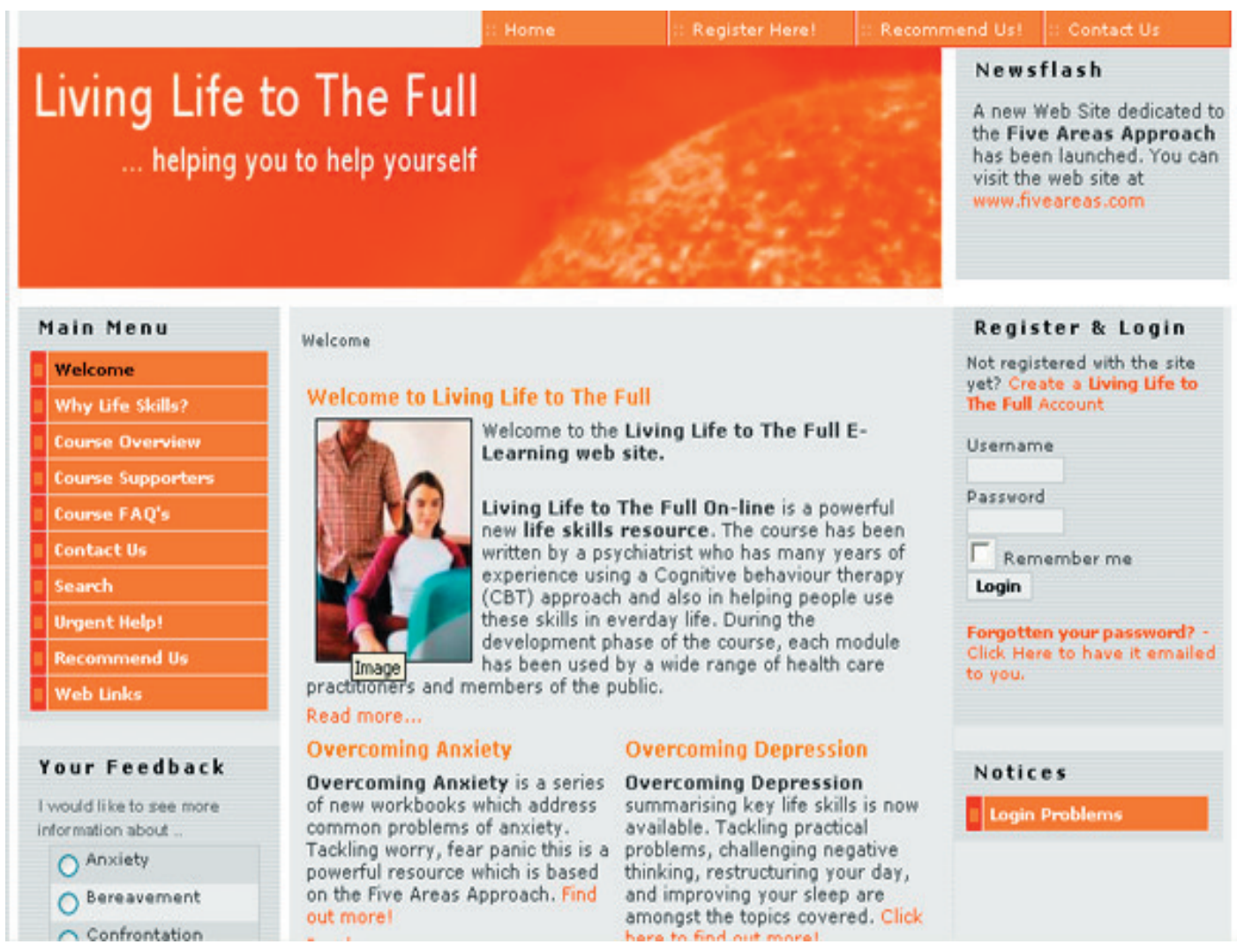

The online sessions include:

- discovering why we feel as we do

- building problem solving skills

- rebalancing relationships

- becoming more active and rediscovering the fun in life

- developing helpful responses to life stress

- discovering how to sleep better

- learning how to change unhelpful and undermining thinking

- focusing on key elements of healthy living

- rating your mood for depression and anxiety

- anxiety control training

- an online self-help forum

In addition, people can sign up for a reminder newsletter to encourage them to use the site.

The site has brought together a number of supporters in the NHS and voluntary sector. Its close links with Depression Alliance Scotland, NHS Greater Glasgow and Clyde, PRIMHe, Triumph over Phobia and National Phobics Society together with the Centre for Change and Innovation at the Scottish Executive Health Department have helped the site develop its profile as a key resource for many people facing low mood and stress. It is increasingly recommended by 
practitioners as somewhere people can go to obtain high quality information in order to help them find ways of moving forward.

b) Facilitated Live chats at www.livinglifetothefull.com: Staff from the Glasgow Condition Management team have facilitated a series of live web chats on www.livinglifetothefull.com centring on return to work issues. These now occur as a regular monthly Health and Employability feature of the website in the afternoon on the first Wednesday of every month and is a resource open to all. Two CMP practitioners work from two computer terminals in the same room to respond to questions. To avoid confusion, one responds to one user's questions/comments to make sure that no-one's questions are overlooked.

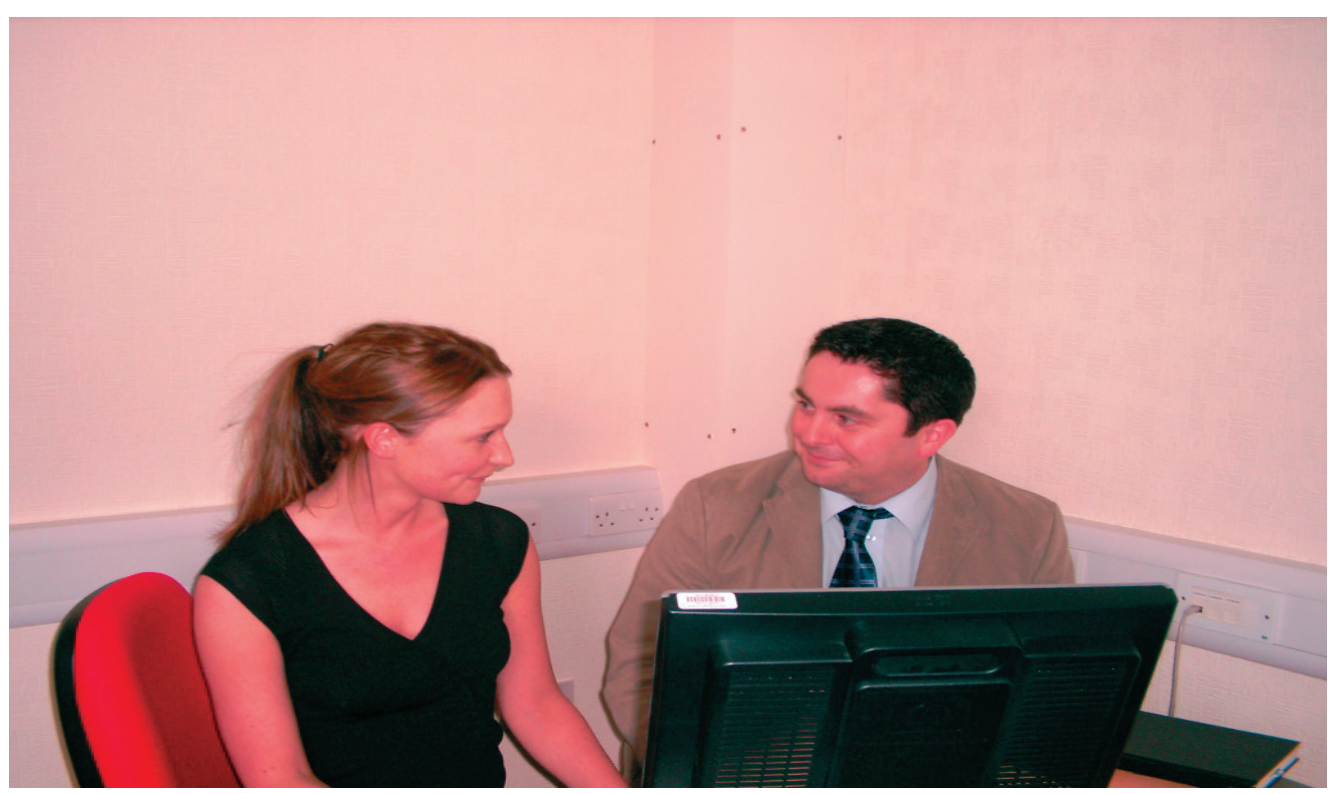

Tommy and Lynne (CMP team leads) supporting a live chat

The CMP Health and Employability live chats have been popular with Living Life to the Full users, some of whom utilised the chat in seeking support for remaining in their current employment and others for rejoining the employment market. The staff who facilitate the web chat have attended the Five areas Condition Management training and are able to offer support and encouragement to users experimenting with CBT self help techniques.

The live chat typically contains between 6 - 15 people, and has received very positive feedback.

A dedicated Health and Employability forum is being launched in October 2007 and in addition to offering support and moderation from experienced condition management practitioners it allows LLTTF users to offer support and advice to each other. These developments allow the same information to be delivered in various formats allowing users preferences to dictate how best they receive support. 


\section{Box 1: A Recent conversation from the LLTTF Condition Management live} online chat:

CMP practitioner Says: When are you due to return to work?

User Says: I don't know when but my psychiatrist has recommended asking for a couple of mornings a week to help get back on track.

CMP practitioner Says: You are, however, in the chat room today, so that is a positive start to not avoiding it

User Says: Yes, l'Il have to do it now!

CMP practitioner Says: Is a couple of mornings a week something you think your employer would support.. have you discussed with your line manager?

User Says: The occupational health $\mathrm{Dr}$ has already mentioned a graduated return.

CMP practitioner Says: Sounds a good idea, cos allows you to phase your return back into work- small steps forwards

User Says: I'm lucky my organisation has this opportunity.

CMP practitioner Says: Excellent that you have Occ health support in your workplace- that should help the process

CMP practitioner Says: Have you set a timescale for returning? what are your thoughts about going back to work?

Edited text (personally identifiable information)

User Says: Timescale? Not yet but I see Occ Health on the XXth and figured I'd go from there. I'm apprehensive as I know I won't be going back to the role I left.

CMP practitioner Says: What will be different about your new role?

Continues:

b). The Living Life to the Full teaching course: an 8-session course of 2 hours per session has been created and is available via admin@ @ivinglifetothefull.com. The course is based around the same course materials as the website and the Overcoming depression and low mood workbook materials (Williams 2006) but is offered as a psycho-educational course for between 10-20 people. Lecture style presentations, small group discussion and "putting into practice tasks" are offered. The course has to date been delivered in both health and community settings (Further Education Colleges in Glasgow and the surrounding area). Attendees have rated the course highly and there have been significant and 
positive changes in mental health literacy (the person's knowledge and selfperceived skills in the causes and self-management of low mood and stress).

c). A free online TV course available via www.livinglifetothefull.com in the registered users area incorporates high quality TV programmes addressing the same life skills modules (assertiveness problem solving, changing negative thinking etc.).

It is important to note that each of these resources is designed to be offered with guidance and support and it is clear that in providing CBT self-help approaches results are far better where support, monitoring and encouragement are provided. (Gellatly et al 2007)

\section{Discussion}

The Five Areas approach provides a common language, model and framework that are accessible and acceptable to a diverse range of multidisciplinary staff. The range of resources available (written workbooks, websites, and on-line TV and chat) increases the support available to the course participant. The results demonstrate the training made a positive impact on staff learning - however crucially the key area of objective skills change only increased significantly in the slow track participants. The major difference between the two training approaches was the time allowed for attendees to consolidate their knowledge and try out and discuss the things they were learning in real life practice. This is an interesting result considering the pressure to deliver training in formats that are easily accessible and acceptable to clinicians. They also emphasise the need for supportive structures and ongoing supervision to be in place. This is an important finding. As a caveat, it should be noted that this analysis reflects only an evaluation at the baseline and immediately at the end of training. It may well be therefore that objective skills rise in slow track participants also in the weeks after the course as they are able to try out and discuss what they have learned within a supervisory framework.

The results also demonstrate that the training is highly satisfactory to multidisciplinary groups of staff. The course and working model therefore appears to make a positive impact on the attitudes, knowledge and skills of diverse groups of staff working with challenging 'more difficult to help' people. The course is best delivered over time and embedded in real life work whilst providing a structure for practice, review and reflection.

\section{Acknowledgements}

The picture of CMP practitioners is of Lynne McAdam and Thomas Forrest of Greater Glasgow and Clyde CMPs. Images are reproduced with permission. Declaration of Conflict of Interest: Dr Chris Williams is author of the LLTTF website and Overcoming depression and low mood workbooks. 


\section{References}

Anderson, L., Lewis, G., Araya, R., Elgie, R., Harrison, G., Proudfoot, J., Schmidt, U., Sharp, D., Weightman, A. \& Williams, C. 2005. Self-help books for depression: How can practitioners and patients make the right choice? British Journal of General Practice 55: 387-392.

Barnes, H. \& Hudson, M. 2006. Pathways to Work: Qualitative Research on the Condition Management Programme. Department for Work and Pensions. Research Report No 346, Corporate Document Services.

Blyth, B. 2006. Incapacity Benefit Reforms: Pathways to Work Pilots Performance and Analysis. Department for Work and Pensions. Working Paper No 26, Corporate Document Services.

Gellatly, J., Bower P, Hennessy S, Richards, D., Gilbody, S. \& Lovell, K. What makes self-help interventions effective in the management of depressive symptoms? Meta-analysis and meta-regression. Psychological Medicine 2007 11: 1-24.

Lovell, K. \& Richards, D. 2000. Multiple access points and levels of entry (Maple): Ensuring choice, accessibility and equity for CBT services. Behavioural \& Cognitive Psychotherapy. 28: 379-391.

Martinez, R., Whitfield, G., Dafters, R. \& Williams, C.J. (in press). Can people read self-help manuals for depression? A challenge for the stepped care model and book prescription schemes. Behavioural \& Cognitive Psychotherapy.

National Institute for Clinical Excellence (NICE) 2004. CG23 Depression: Management of Depression In Primary and Secondary Care; www.nice.org.uk

Williams, 2006. Overcoming Depression and Low Mood, 2nd edition. Hodder Arnold: London.

Williams, C. \& Garland, A. 2002. A cognitive-behavioural therapy assessment model for use in everyday practice. Advances in Psychiatric Treatment 8: 172 179.

Williams, C.J., Morrison, J., Wilson, P., Whitfield, G., Walker, A., McMahon, A. \& Wallace, I. 2007. An Evaluation of the Effectiveness of Structured Cognitive Behaviour Therapy Self-Help Materials Delivered by a Self-Help Support Worker within Primary Care. CZH/4/61 CSO Final submitted report. 